\title{
UVCS Observations of a Helical CME Structure
}

\author{
R. M. Suleiman ${ }^{1,2}$, N. U. Crooker ${ }^{2}$, J. C. Raymond ${ }^{1}$ \\ and A. van Ballegooijen ${ }^{1}$ \\ ${ }^{1}$ Harvard-Smithsonian Center for Astrophysics, 60 Garden Street, Cambridge, MA 02138, USA \\ ${ }^{2}$ Center for Space Physics, Boston University, 725 Commonwealth Avenue, Boston, MA 02215, \\ USA
}

\begin{abstract}
.
A helical structure in the coronal mass ejection (CME) of 12 September 2000 was observed by the Ultraviolet Coronagraph Spectrometer (UVCS) aboard the Solar and Heliospheric Observatory $(\mathrm{SOHO})$ at heliocentric distances of 3.5 and $6 \mathrm{R}_{\odot}$. A difference of $300 \mathrm{~km} \mathrm{sec}^{-1}$ in line-of-sight velocities for two segments of the helix obtained from Doppler measurements implies expansion and allows one to distinguish which segment was closest to the observer. The tilt of the segment then determines the handedness. Observed Ly $\alpha$ and C III line emissions indicate that the helix was threaded with filament plasma of varying density. While the helix constituted the bright core of filament plasma, the helix itself was most likely not the pre-existing filament structure.
\end{abstract}

Keywords. Sun: corona, Sun: coronal mass ejections (CMEs), Sun: filaments, Sun: UV radiation

\section{Introduction}

In this paper we use the observations by instruments aboard SOHO to study a helical structure in a CME that occured on 12 September 2000. In particular, we use the Doppler shift of UVCS (Kohl et al. 1995) to derive its handedness. In addition, we combine the white-light data of LASCO C2 and C3 (Brueckner et al. 1995) and the UVCS spectroscopic observations to address questions about the morphology of filament plasma.

\section{Analysis}

\subsection{Spectroscopic Observations}

The Ultraviolet Coronagraph Spectrometer (UVCS) on the SOHO spacecraft was wellpositioned to observe a complicated CME on 12 September 2000. Observations were made from the O VI spectrometer channel, which is optimized to observe the O VI $\lambda \lambda 1032,1037$ doublet and the H I $\lambda 1026$ (Ly $\beta$ ) line and covers the spectral range 945-1123 $\AA$ (473-561 $\AA$ in second order). The O VI includes a redundant Ly $\alpha$ path which has a convex mirror between the grating and the O VI detector that focuses the H I $1216 \AA$ (first order) onto the O VI channel.

In the 12 September observations, the Si XII $\lambda 1041.26$ and $\mathrm{Mg}$ X $\lambda 1219.7$ lines are not present which implies that the temperature of the plasma is significantly less than $10^{6} \mathrm{~K}$.

The density of this cold plasma (e.g., plasma emitting at C III $\lambda 977$; Akmal et al. 2001) was calculated using a commonly used estimate of the emission measure:

$$
\int n_{e} n_{H} d h=I_{\lambda} /\left\langle P_{\lambda}\right\rangle c m^{-5}
$$




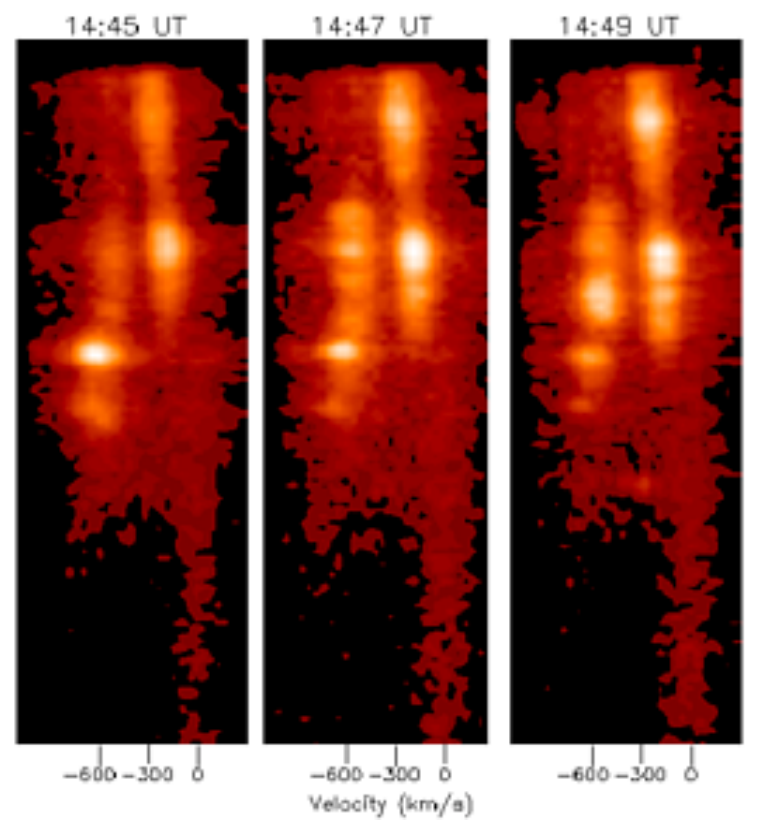

Figure 1. Spectral distribution of the Ly $\alpha$ line at heliocentric height of $6 \mathrm{R}_{\odot}$, which shows the Doppler shift. Each panel respents 2 minutes of observation. A Doppler shifts of $300 \mathrm{~km} \mathrm{sec}-1$ and $600 \mathrm{~km} \mathrm{sec}^{-1}$ can be easily seen.

where $\mathrm{I}_{\lambda}$ is the intensity of the spectral line, $\left\langle\mathrm{P}_{\lambda}\right\rangle$ is the radiative loss for a given line, $h$ is distance along the line-of-sight, and $n_{e}$ and $n_{H}$ are the electron and hydrogen densities. The radiative losses for the lines used in determining the emission measure are obtained from an updated version of Raymond \& Smith (1977) code. The densities in the C III emitting plasma exceed $3 \times 10^{5} \mathrm{~cm}^{-3}$, and the ionization temperature ranges from below $25,000 \mathrm{~K}$ to above $150,000 \mathrm{~K}$. This is the ionization temperature in ionization equilibrium. Although there is no good reason to believe that equilibrium was reached in this case, but based on the excitation rates the temperature cannot be $<25,000 \mathrm{~K}$ and based on the observed line widths it cannot be $>150,000 \mathrm{~K}$.

Of particular interest were the Doppler shifts for the Ly $\alpha$ and C III lines. Doppler shifts give line-of-sight velocities of the erupting plasma. The velocity Doppler shift is governed by the usual formula $\Delta \lambda / \lambda=v / c$, for collisionally excited lines. For lines excited by radiation scattering, the wavelength shift can be smaller because the profile is weighted by the emission line profile from the solar disk (Noci \& Maccari 1999). Due to the high velocities of the CME material, the radiation scattering components are severely Doppler dimmed (Withbroe et al. 1982), and the lines are mostly collisionally excited.

Examples of UVCS Doppler shift measurements from the 12 September CME are given in Figure 1. They show spectral images of the Ly $\alpha$ line at three different times. The vertical axis represents position along the UVCS slit and the horizontal axis is the wavelength, in this case converted to velocity. A Doppler shifts of $300 \mathrm{~km} \mathrm{sec}^{-1}$ and 600 $\mathrm{km} \mathrm{sec}^{-1}$ can be seen in all three panels.

In contrast to Figure 1, the two right panels in Figure 2 give spatial rather than wavelength information. They show Ly $\alpha$ and C III $\lambda 977$ intensity maps as a function of time at $6 \mathrm{R}_{\odot}$. The UVCS slit was positioned directly south of the Sun (at position angle (PA) 180 degrees South). The vertical axis shows time increasing from the bottom, which converts to radial distance under the assumption of constant flow speed. The horizontal 


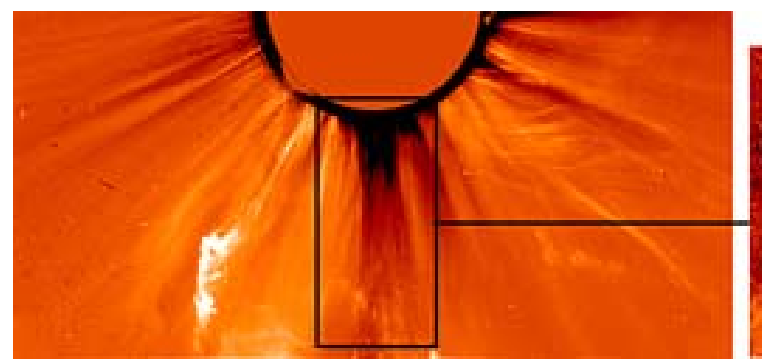

CIII977 A

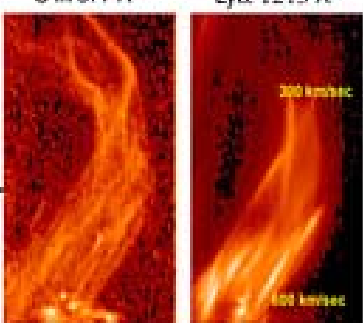

Figure 2. Ly $\alpha$ and C III line intensity maps along the UVCS slit (horizontal axis) as a function of time (vertical axis) at heliocentric height of $6 \mathrm{R}_{\odot}$ (right panel). The left panel shows LASCO C2 image with UVCS slit superimposed

axis is the spatial coordinate in arcmin along the entrance slit. The panels cover three hours of observations, from 14:45 UT to 17:45 UT. At 14:45 UT the Doppler shift is $600 \mathrm{~km} \mathrm{sec}^{-1}$ while later 16:45-17:45 UT the Doppler shift is $300 \mathrm{~km} \mathrm{sec}^{-1}$. The shift of plasma from east to west and then to the east along the UVCS slit implies helical structure for $\sim 3$ hours in both lines. Combined with Doppler shifts, we deduce that the portion of the helix tilted upward east to west is the left leg of the flux rope.

The two values of Doppler shift imply that the helix is expanding. Further, the faster Doppler shift of $600 \mathrm{~km} \mathrm{sec}^{-1}$ must apply to the near side of the helix tilting to the West, while the slower shift of $300 \mathrm{~km} \mathrm{sec}^{-1}$ must apply to the far side of the helix, tilting to the East. This is counter-clockwise for an observer looking radially out from the Sun, implying a right-handed helix. Thus Doppler shifts can uniquely determine the handedness of a helical structure.

To gain an understanding of the context of the observed plasma, we combine the spectroscopic observations with LASCO C2 white light images. In this way we can identify the location of the plasma with respect to the 3-part CME structure. The left panel of Figure 2 is the LASCO C2 image at 14:45 UT with the region corresponding to the UVCS observations superimposed. The vertical side of the box represents the distance that the plasma traveled in three hours, where the horizontal side represents the field of view of the UVCS slit. The plasma traveled at a speed of $\sim 390 \mathrm{~km} \mathrm{sec}^{-1}$. If the plasma structure observed in the UVCS panels were advecting with the flow and undergoing no dynamic evolution, the structure in the rectangle on the LASCO panel would be the same as that observed by UVCS. Since the helical structure in the UVCS panel is not apparent in the LASCO panel, we conclude that dynamic evolution over the 3 -hour interval obscured an exact match. Over the shorter interval at $3.6 \mathrm{R}_{\odot}$ in Figure 3 , however, an excellent match is obtained.

Figure 3 corresponds to 32 minutes of observations, starting at 13:28 UT and ending at 14:00 UT. The structure in the UVCS data, especially that of the C III line, is a remarkable match to the structure seen by LASCO C2. The helical form is more pronounced in the Ly $\alpha$ compared to the $\mathrm{C}$ III line. On the other hand, compared to Figure 2 at $6 \mathrm{R}_{\odot}$, the helical structure in the $\mathrm{C}$ III line at $3.6 \mathrm{R}_{\odot}$ is less pronounced. This is due to the fact that the observation at $3.6 \mathrm{R}_{\odot}$ is significantly shorter than that at $6 \mathrm{R}_{\odot}$, as pointed out above.

Also as pointed out above, the C III emission indicates cool material $(<150,000 \mathrm{~K})$ typical of a filament. However, the location of some of this cool plasma, as clearly seen by Figure 3, is at the edge of what appears to be a tightly wound flux rope, beyond most of the more loosely wound helical structure apparent in the UVCS data in Figure 3. The tightly wound flux rope is the type thought to be generated by reconnection of 


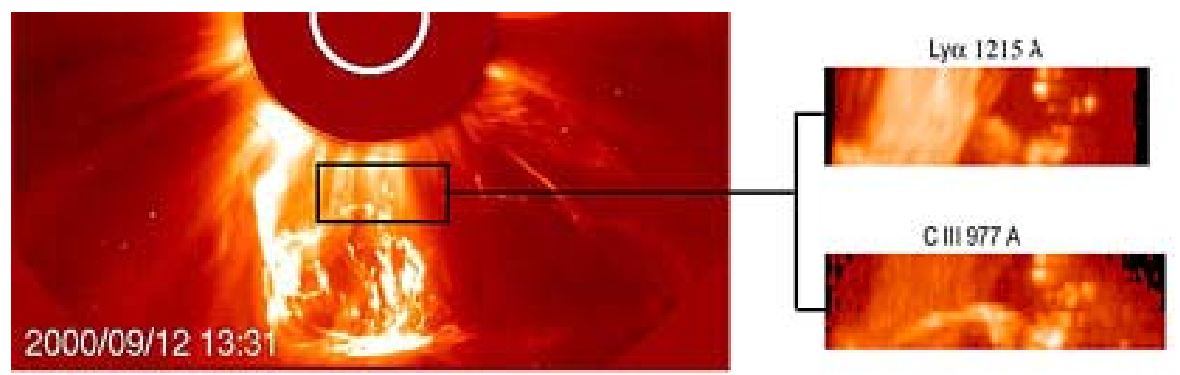

Figure 3. Ly $\alpha$ and C III line intensity maps along the UVCS slit (horizontal axis) as a function of time (vertical axis) at heliocentric height of $3.6 \mathrm{R}_{\odot}$ (right panel). The left panel shows LASCO C2 image with UVCS slit superimposed.

coronal arcade loops and thus should contain hot plasma. Evidence of this reconnection is a concurrent, long lasting arcade event in EIT data (e.g., Vršnak et al. 2003).

\section{Discussion}

We have presented an analysis of a helical structure observed by UVCS/SOHO in the 12 September 2000 CME. The UVCS Doppler shift observations can distinguish field lines in the near side of the helix from those on the far side and consequently the skew of these fields and the handedness of what appeared to be a portion of a flux rope in the CME. We conclude that the helix is right-handed.

The analysis suggests that the cool plasma of the filament appeared in the newlyformed helix of the CME which is usually associated with the dark cavity of 3-part CME structure rather than the bright core where current understanding of filaments would suggest. Basic reconnection through the current sheet ( Lin \& Forbes 2000), which presumably creates the helix, is generally thought to allow only ambient very hot ( $>$ million $\mathrm{K}$ ) coronal plasma to travel upward into the helix. A possible explanation of the apparent contradiction is that the cooler filament plasma somehow became caught up in this reconnection and gained access to the helix by losing its magnetic coherence. A more interesting possibility is that the field lines carrying filament material remained coherent but moved from the axis of the flux rope to the edge by an internal kink instability. In future work we will explore these possibilities in detail.

\section{Acknowledgements}

R. M. S. thanks the Scientific Organizing Committee for travel support to the IAU 226 symposium. This work was supported by NASA grant NNG04GE84G to the Smithsonian Astrophysical Observatory and NSF grant ATM-0119700 to Boston University. The authors would like to thank A. Vourlidas for the LASCO data and L. Strachan for useful discussions.

\section{References}

Akmal, A., Raymond, J. C., Vourlidas, A., Thompson, B., Ciaravella, A., Ko, Y.-K., Uzzo, M., \& Wu, R. 2001, ApJ 553, 922

Brueckner et al. 1995, Sol. Phys. 162, 357

Kohl, J. L., et al. 1995, Sol. Phys. 162, 313

Lin, J., \& Forber, T.G. 2000, J. Geophys. Res. 105, 2375

Noci, G., \& Maccari, L. 1999, Astron. Astrophys. 341, 275-285

Raymond, J.C., \& Smith B.W. 1977, ApJ 35, 419 
Withbroe, G.L., Kohl, J.L., Weiser, H., \& Munro, R.H. 1982, Space Sci. Rev. 33, 17

Vršnak, B., Warmuth, A., Maricic, Otruba, W., \& Ruzdjak, V. 2003, Sol. Phys. 217, 187

\section{Discussion}

Kouтснму : UVCS reconstructed images taken in Ly $\alpha$ and C III look quite different from images seen on W-L, Do you have any comment on that?

SulEIMAn: This is true only in the UVCS observations at $6.0 \mathrm{R}_{\odot}$, The UVCS observation at $6.0 \mathrm{R}_{\odot}$ was for over $3 \mathrm{hrs}$. The dynamics of the CME changed and thus the helical structure was not evident in LASCO/C2 data but it shows the location of the leg of the flux rope. In the case of UVCS observations at $3.6 \mathrm{R}_{\odot}$, there was a very remarkable match between UVCS and LASCO/C2 data. As a matter of fact UVCS was able to provide temperature and density information about the CME. In this case UVCS showed that the filament plasma resided on the edge of the flux rope rather than at the axis of the flux rope. This is a very important piece of observation since CME models predict that filament material should be at the axis of the flux rope. The remarkable match between UVCS and LASCO/C2 at 3.6 $\mathrm{R}_{\odot}$ is very clear since the time period of the observation is $32 \mathrm{~min}$, comparing to over $3 \mathrm{hrs}$ at $6 \mathrm{R}_{\odot}$ where the dynamics of the CME have changed.

BothmeR: 1. Handedness of flux rope overlying sinistral filament?

2. The analyzed magnetic structure is not the flux rope but expanded prominence (prominence is base of overlying flux rope).

SulEiman: 1. The handedness of the overlying arcade has an inverse relationship with the chirality of the filament. Since the filament is sinistral then the overlying arcade is right skewed.

2. The flux rope did not exist prior to the eruption but was formed later on as part of the CME liftoff process. If the flux rope did exist prior to eruption then the filament channel would have disappeared, after the eruption, which $\mathrm{H} \alpha$ observations do not show. In addition the EIT observations show the appearance of the overlying arcade as the filament disappears in EIT images. 